\title{
Myocardial perfusion imaging and coronary calcium score: A marriage made in heaven
}

\author{
Nataliya Pyslar, MD, a and Rami Doukky, MD, MSc, FASNC ${ }^{a, b}$ \\ a Division of Cardiology, Cook County Health, Chicago, IL \\ b Division of Cardiology, Rush University Medical Center, Chicago, IL
}

Received Nov 12, 2019; accepted Nov 12, 2019

doi: 10.1007/s12350-019-01966-8

\section{See related article, pp. 2086-2096}

A large body of evidence has been generated in support of the use of myocardial perfusion imaging (MPI), in the diagnostic and prognostic assessment of patients with suspected coronary artery disease (CAD). ${ }^{1-5}$ In principle, stress MPI is best suited for patients with intermediate likelihood of CAD. However, over the past two decades, the prevalence of ischemia in patients without established CAD has dramatically declined. ${ }^{6}$ Therefore, as patients with lower probability of CAD are being tested, the prognostic and diagnostic utility of stress MPI is expected to be diminished. ${ }^{3,7,8}$ Importantly, MPI provides no insight into non-flow limiting plaques. On the other hand, the coronary artery calcium score (CACS) is an excellent anatomic measure of atherosclerotic plaque burden and is a well-established risk predictor. ${ }^{9,10}$ CACS of zero is a powerful negative risk marker with a long-term warranty period in asymptomatic patients. Although non-calcified plaque is observed in $1 \%-2 \%$ of symptomatic patients with CACS of 0 , their prognosis remains excellent. ${ }^{11}$ In contrast, Agatston CACS $\geq 1,000$ is associated with high hazard of cardiovascular events approaching the risk of patients with established CAD. ${ }^{12}$ Engbers et al demonstrated that the frequency of an abnormal MPI was greater in patients with high CACS, as patients with a CACS $\geq 1000$ were roughly five times more likely to have abnormal MPI compared with those with CACS of zero.

Reprint requests: Rami Doukky, MD, MSc, FASNC, Division of Cardiology, Cook County Health, Chicago, IL; rami_doukky@ rush.edu

J Nucl Cardiol 2021;28:2097-9.

$1071-3581 / \$ 34.00$

Copyright (C) 2019 American Society of Nuclear Cardiology.
In fact, CACS $\geq 1,000$ appeared to be an even greater predictor for future events than a large perfusion defect (HR 7.57 vs 3.74, respectively). While there is evident association between increasing CACS and abnormal MPI, each represents a different aspect of the disease process; i.e. coronary atherosclerosis versus myocardial perfusion. ${ }^{13}$ Importantly, CACS cannot reliably serve as a diagnostic tool for obstructive CAD in symptomatic subjects, as the correlation between CACS and luminal stenosis is poor. ${ }^{13}$ On the other hand, while MPI has its shortcomings, CACS can identify subclinical or extensive disease in patients with suspected CAD. Clearly, CACS and MPI provide complementary information and it is only logical to leverage their combined strengths. Indeed, with the widespread use of hybrid SPECT/CT and PET/CT, it is possible to acquire both MPI and CACS in a single session. The addition of CACS to MPI in patients undergoing SPECT/CT or PET/CT only adds 1-2 minutes of scan time, minimal cost, and 1.0$1.5 \mathrm{mSv}$ effective dose of radiation exposure. The question is whether combining MPI and CACS data will result in an incremental diagnostic and prognostic value. Moreover, how can we practically integrate MPI and CACS data to guide subsequent treatment decisions?

In this issue of the journal, Sharma et al analyzed 655 symptomatic patients who underwent pharmacologic stress SPECT-MPI and CACS and were followed for a mean of 2.5 years for all-cause death, myocardial infarction, and coronary revascularization. ${ }^{14}$ As was observed in prior studies, ${ }^{13}$ the magnitude of CACS correlated with the frequency of abnormal MPI (total perfusion deficit $>5 \%$ ). The authors identified a CACS of $\geq 216$ as a critical threshold with optimal discriminative capacity, which was an independent predictor of mortality. Based on four possible combinations of MPI finding (normal vs abnormal) and CACS status $(<216$ vs $\geq 216$ ), the authors demonstrated that those with MPI+/CACS + have the highest mortality rate $(12.5 \%)$ and those with MPI-/CACS - scans have the lowest 
mortality $(1.6 \%)$, while patients with MPI-/CACS+ and MPI $+/ C A C S$ - represented intermediate risk groups with mortality rates of $10.7 \%$ and $4.8 \%$, respectively. Thus, CACS + cohort were at higher mortality risk despite normal MPI. Additionally, CACS + status was associated with increased revascularization rates regardless of MPI findings. Clearly, the retrospective, single-center design and relatively short follow-up need to be accounted for when interpreting these results. Despite the aforementioned limitations, the study adds to the mounting evidence indicating enhanced prognostic value with concomitant use of CACS and MPI.

Although adding CACS to MPI findings is undoubtedly beneficial, devising a simple method to integrate these variables can be challenging, given the continuous and wide-spectrum nature of CACS data. Sharma et al took a simplistic approach to resolve this dilemma by deriving a critical CACS threshold, based on which they dichotomized their patients into CACS+ and CACS - subgroups. Firstly, the identified CACS threshold of $\geq 216$ cannot be generalized to other populations, as it has not been externally validated. Secondly, a simplistic threshold does not take into account the graded correlation between CACS and outcome. Similarly, a dichotomous classification of MPI to normal vs abnormal does not capture the nuances of MPI abnormalities. Nonetheless, the current study reinforces the fact that adding CACS to MPI data provides complementary prognostic value that can guide subsequent decision making.

Over the past two decades, there have been a number of publications which described similar findings. Chang et al investigated the incremental prognostic value of CACS combined with MPI in an asymptomatic population with risk factors. ${ }^{15}$ Expectedly, the highest event rates occurred among subjects with both high CACS and abnormal MPI. The authors suggested that adding CACS data might refine the "warranty period" provided by a normal MPI. ${ }^{15}$ Additionally, in a cohort of 4,897 symptomatic patients undergoing CACS/SPECT, Engbers et al demonstrated low rate of cardiovascular events in patients with CACS of 0, regardless of MPI finding. ${ }^{13}$ Moreover, in a study of symptomatic patients undergoing PET/CT, Schenker et al reported a wide range of CACS in patients with normal PET-MPI, confirming MPI's limitation in detecting subclinical atherosclerosis. Conversely, the wide distribution of CACS in patients with abnormal PET suggested that CACS cannot reliably serve as a screening tool for obstructive CAD in symptomatic patients. ${ }^{16}$ As was shown by Chang et al, Schenker et al demonstrated a stepwise increase in the risk of cardiac events with increasing CACS regardless of MPI findings. These data indicate that a strategy of combining CACS findings with MPI results, offers improved risk stratification over conventional approaches. ${ }^{16}$

CACS data not only augments the prognostic value of MPI, but often enhances the diagnostic acumen of the test. For example, a CACS of zero may affirm low risk of a "borderline" MPI result due to an artifact or discrepant data between the stress electrocardiography and MPI conclusions. ${ }^{17}$ On the other hand, very high CACS $\geq 1000$ may raise suspicion of multivessel CAD in symptomatic patients with an apparently normal MPI due to "balanced ischemia". Importantly, elevated CACS in patients with normal MPI would prompt an earlier focus on aggressive risk factors modification and initiation of statin and aspirin therapies. This was demonstrated in the SCOT-HEART trial, where evidence of anatomic disease on coronary CT angiography prompted initiation of aggressive medical therapy which translated into improved patients outcomes. ${ }^{7}$

Although it is clear that the roles of MPI and CACS are complementary, combining these parameters in a manner that can guide dichotomous decision-making is rather challenging, given the wide spectrum of CACS data and the complexity of MPI interpretation. Integrating clinical and ancillary MPI findings, such as transient ischemic dilation, ejection fraction, stress variables, etc., only adds to this complexity. Indeed, it may be impossible for the human brain to integrate such a wide range of clinical and imaging variables. Here, there is a distinct role for artificial intelligence technologies, particularly machine learning, which may provide future solutions to this challenge. In a recent multicenter study of 2,619 patients who underwent SPECT-MPI and followed for 3 years, Betancur et al demonstrated that a machine learning algorithm encompassing 47 variables (22 imaging, 8 stress test, 17 clinical) provided a significant improvement in predictive accuracy for major adverse cardiac events when compared with expert readers, automated quantitative analysis, and a machine learning algorithm relying on imaging data alone. ${ }^{18}$ The study was the first to report improved prognostic accuracy using machine learning algorithms applied specifically to nuclear cardiac imaging. The concept of incorporating clinical, stress, and imaging parameters in complex machine learning models, can be easily extended to include CACS to further enhance risk prediction. Full integration of these variables in imaging, reporting, risk prediction, and decision-making will cement a lasting complementary partnership between MPI and CACS. ${ }^{19,20}$

\section{Disclosures}

Dr. Rami Doukky received Research Grant funding from Astellas Pharma Global Development (Northbrook, IL). Dr. Nataliya Pyslar has no conflicts to disclose. 


\section{References}

1. Cantoni V, Green R, Acampa W, Zampella E, Assante R, Nappi C, et al. Diagnostic performance of myocardial perfusion imaging with conventional and CZT single-photon emission computed tomography in detecting coronary artery disease: A meta-analysis. J Nucl Cardiol Off Publ Am Soc Nucl Cardiol 2019;14:1-8.

2. Doukky R, Frogge N, Balakrishnan G, Hayes K, Collado FM, Rangel MO, et al. The prognostic value of cardiac SPECT performed at the primary care physician's office. J Nucl Cardiol 2013;20:519-28.

3. Doukky R, Hayes K, Frogge N, Balakrishnan G, Dontaraju VS, Rangel MO, et al. Impact of appropriate use on the prognostic value of single-photon emission computed tomography myocardial perfusion imaging. Circulation 2013;128:1634-43.

4. Iskander F, Iskander M, Gomez J, Doukky R. Prognostic value of regadenoson stress myocardial perfusion imaging in patients with left bundle branch block or ventricular paced rhythm. J Nucl Cardiol 2019. https://doi.org/10.1007/s12350-019-01762-4.

5. Hachamovitch R, Berman DS, Kiat H, Cohen I, Cabico JA, Friedman $\mathrm{J}$, et al. Exercise myocardial perfusion SPECT in patients without known coronary artery disease: Incremental prognostic value and use in risk stratification. Circulation 1996;93:905-14.

6. Rozanski A, Gransar H, Hayes SW, Min J, Friedman JD, Thomson LE, et al. Temporal trends in the frequency of inducible myocardial ischemia during cardiac stress testing: 1991 to 2009. J Am Coll Cardiol 2013;61:1054-65.

7. SCOT-HEART Investigators. CT coronary angiography in patients with suspected angina due to coronary heart disease (SCOT-HEART): An open-label, parallel-group, multicentre trial. Lancet (Lond Engl) 2015;385:2383-91.

8. Neglia D, Rovai D, Caselli C, Pietila M, Teresinska A, AguadeBruix S, et al. Detection of significant coronary artery disease by noninvasive anatomical and functional imaging. Circ Cardiovasc Imaging 2015;8:e02179.

9. Budoff MJ, Mayrhofer T, Ferencik M, Bittner D, Lee KL, Lu MT, et al. Prognostic value of coronary artery calcium in the PROMISE Study (Prospective Multicenter Imaging Study for evaluation of chest pain). Circulation 2017;136:1993-2005.

10. Greenland P, Blaha MJ, Budoff MJ, Erbel R, Watson KE. Coronary calcium score and cardiovascular risk. J Am Coll Cardiol 2018;72:434-47.

11. Mittal TK, Pottle A, Nicol E, Barbir M, Ariff B, Mirsadraee S, et al. Prevalence of obstructive coronary artery disease and prognosis in patients with stable symptoms and a zero-coronary calcium score. Eur Heart J Cardiovasc Imaging 2017;18:922-9.

12. Blaha MJ, Mortensen MB, Kianoush S, Tota-Maharaj R, CainzosAchirica M. Coronary artery calcium scoring: Is it time for a change in methodology? JACC Cardiovasc Imaging 2017;10:92337.

13. Engbers EM, Timmer JR, Ottervanger JP, Mouden M, Knollema $\mathrm{S}$, Jager PL. Prognostic value of coronary artery calcium scoring in addition to single-photon emission computed tomographic myocardial perfusion imaging in symptomatic patients. Circ Cardiovasc Imaging 2016;9:e003966.

14. Sharma V, Mughal L, Dimitropoulos G, Sheikh A, Griffin M, Moss A, et al. The additive prognostic value of Coronary Calcium Score (CCS) to Single Photon Emission Computed Tomography Myocardial Perfusion Imaging (SPECT-MPI)-real world data from a single centre. J Nucl Cardiol 2019. https://doi.org/10.1007/s12 350-019-01965-9

15. Chang SM, Nabi F, Xu J, Peterson LE, Achari A, Pratt CM, et al. The coronary artery calcium score and stress myocardial perfusion imaging provide independent and complementary prediction of cardiac risk. J Am Coll Cardiol 2009;54:1872-82.

16. Schenker MP, Dorbala S, Hong EC, Rybicki FJ, Hachamovitch R, Kwong RY, et al. Interrelation of coronary calcification, myocardial ischemia, and outcomes in patients with intermediate likelihood of coronary artery disease: A combined positron emission tomography/computed tomography study. Circulation 2008;117:1693-700.

17. Doukky R, Nigatu A, Khan R, Anokwute C, Fughhi I, Ayoub A, et al. Prognostic significance of ischemic electrocardiographic changes with regadenoson stress myocardial perfusion imaging. $\mathrm{J}$ Nucl Cardiol 2018. https://doi.org/10.1007/s12350-018-1415-4.

18. Betancur J, Otaki Y, Motwani M, Fish MB, Lemley M, Dey D, et al. Prognostic value of combined clinical and myocardial perfusion imaging data using machine learning. JACC Cardiovasc Imaging 2018;11:1000-9.

19. Gomez J, Doukky R. Artificial intelligence in nuclear cardiology. J Nucl Med 2019;60:1042-3.

20. Tilkemeier PL, Bourque J, Doukky R, Sanghani R, Weinberg RL. ASNC imaging guidelines for nuclear cardiology procedures: standardized reporting of nuclear cardiology procedures. J Nucl Cardiol 2017;24:2064-128.

Publisher's Note Springer Nature remains neutral with regard to jurisdictional claims in published maps and institutional affiliations. 